\title{
Impact of Experimenting Computational Statistics for Data Science in Introductory Statistics Course: A Malaysian Perspective
}

\author{
Kathiresan Gopal $^{1, *}$, Nur Raidah Salim ${ }^{1}$, Ahmad Fauzi Mohd Ayub ${ }^{1,2}$ \\ ${ }^{1}$ Institute for Mathematical Research, Universiti Putra Malaysia, 43400 UPM Serdang, Malaysia \\ ${ }^{2}$ Faculty of Educational Studies, Universiti Putra Malaysia, 43400 UPM Serdang, Malaysia
}

\begin{abstract}
The conventional teaching methods for statistics, especially for introductory statistics course (precursor to data science) are not in accordance with the advancement and demand of today's data-centric world. As the backbone of modern data science, computational statistics is deemed to revamp the learning of statistics to a large extent. As such, this study investigated the impact of experimenting computational statistics for data science in introductory statistics course from a Malaysian perspective. We employed a pre-, postand delayed post-test quasi-experimental design in this study. Our sample included 100 randomly selected students enrolled in an introductory course in a Malaysian public university. Students were equally separated into computational (followed computational statistics approach in learning statistics) and conventional (followed only conventional approach in learning statistics) groups. Students in both groups were assessed at three stages: initial, medial and final, respectively. Their performance (assessment marks) was used to measure the effectiveness of computational statistics approach in the learning process of introductory statistics. Results attested that computational group students performed significantly better than the conventional group students in both medial and final assessments. Furthermore, computational group students showed greater improvement from initial to medial assessment and sustained their performance from medial to final assessment, indicating that their knowledge acquisition was effective in the computational statistics approach. Our findings implied that computational statistics approach in introductory statistics course exerted a positive impact on students' statistics learning and performance, leading towards effective knowledge and computing skills acquisition for data science.
\end{abstract}

Keywords Computational Statistics; Data Science; Introduc-tory Statistics; Statistics learning and Performance

\section{Introduction}

As data analytics has become a part and parcel of today's world, aspiring graduates from various disciplines of study need data science to contribute valuably to any modern workplace across the globe. The availability of vast data (big data) require students to understand data using computational tools for data analytics (management, exploratory analysis, visualization, and modelling) [1 - 4]. Data centered approach to problem finding and solving requires students to perceive features of computation and data science which require statistical skills as early as possible [5]. Hence, setting up forerunners in introductory statistics course (precursor to data science) will ensure students to kick-start [6]. Students should learn how to utilize previous outcomes from existing data to perform statistical explorations and to decide on the choice of analysis (opinion of Diane Lambert of Google) [7]. However, in contrast to that, students with practically minimum (or zero) computational experience are not trained to do so [7]. As a consequence, the conventional teaching methods for statistics, especially for introductory statistics course are not in accordance with the advancement and demand of data science [8]. In line with that, several researchers asserted that computational statistics approach (practical data computing) can improve the existing conventional teaching methods for statistics $[1,9,10]$.

\subsection{Problems in Conventional Teaching Methods}

Students usually face a hard time in apprehending the conventional teaching methods of statistics theoretically [11, 12]. This has certainly made the learning of statistics to be difficult generally. One such difficulty can be seen when preliminary statistical concepts like sampling techniques, probability distributions and hypotheses testing are taught by requiring students to work through formulas by hand [13]. Apparently, some statistics instructors still regard the mathematical fundamentals of statistics alone are sufficient for novice students to have an essential understanding of statistics [14]. They failed to realize that to do data analysis is to do computing [14]. Some instructors may argue that by using a calculator is sufficient to 
do computing, nevertheless, calculator is not a complete tool for statistical computation. Although being able to assist in arithmetic calculations, however analysis produced using calculator is not reproducible as it is always a one-off process [15]. Moreover, the computation performed does not help students in developing in-depth understanding of the underlying statistical concepts [14]. Ultimately, calculators may only be adequate for datasets in textbooks but working with large empirical data is not realistic, as data are not provided as persistent objects to students [16].

\subsection{The Computational Statistics Approach}

The simplest manifestation of computing in statistics learning is statistical computing. Through this approach, the course is taught as usual but statistical software are primarily used as computational tools to replace by-hand calculations and manual plotting [17]. Statistical computing helps to speed up calculations (especially for large datasets) and to produce welldesigned plots. The proper approach of teaching introductory statistics (towards data science) is undoubtedly computational statistics [8]. Computational statistics is verily the central component of data science i.e. to answer questions using data and to convey the mitted results [12]. Some fundamental changes to the contents of the course occur in this approach. Essentially, the availability of statistical software as computing resource determines how the teaching is done [17]. In any case, it is inevitable to engage students in at least preliminary level of statistical computing [18]. As such, the common practice in most statistics courses is to combine aspects of both approaches [19]. The proportion of aspects used from each approach depends on factors such as aim of the course, resources of technology and preference of the instructor [18]. However, in the Malaysian context, statistical computing is often more emphasized, in which students are exposed to statistical software on a minimal scale [20]. Through this practice, Malaysian students do not get properly trained in computational statistics and their path to data science is obscured.

\subsection{RStudio as a computational statistics tool}

The open source statistical environment and programming language, $\mathrm{R}$ has become the de-facto standard for data science [21]. R was ultimately created with statistics and data in mind [22]. Almost any statistical techniques and tests are readily available in $\mathrm{R}$, making it the best choice for data science (or data analysis) [23]. $\mathrm{R}$ is relevant beyond classroom learning as it complies with any type and level of users; and powerful, flexible, and extensible [24]. The development of RStudio has made $\mathrm{R}$ even easier to get started with, and more accessible. RStudio is an open-source interface and integrated development environment (IDE) for R [25]. RStudio was built exclusively for $\mathrm{R}$ and also to expand the power of $\mathrm{R}$, which allows integration of $\mathrm{R}$ with other computational tools as well [26]. From now on, the term RStudio will be used to denote both $\mathrm{R}$ and RStudio respectively.

The primary advantage of using RStudio is it enables students to perform data analysis, computations and create data visualizations by writing scripts and coding; and not by pointing and clicking or choosing analysis technique from a dropdown menu (which is common in commercial statistical software) [27]. Students do not work in a black-box environment but get an interactive experience that encourages experimentation; exploration and play when using RStudio [24]. This process may sound intimidating for students who are new to computer programming, yet this is where students really get to learn and deeply understand the underlying statistical concepts [28]. Although initially not designed as a teaching tool, RStudio has the potential to become one as it allows students to think, reason and learn with data [26]. Introductory statistics course can be a promising platform to provide training in RStudio, as it is slowly becoming a norm for some instructors to include the applications of RStudio in their lectures too [29].

One prominent effort of integrating RStudio in introductory statistics course was initiated by a project called MOSAIC, which is a group of instructors collaborating to come up with innovative ways to intro-duce mathematics, statistics, computation, and modelling to college and university students [30]. The aim of MOSAIC is to disseminate ideas and resources to enhance existing instruction methods and to develop a curricular and an evaluation plan for sharing and assessment purposes. Additionally, its goal is to create an extensive way for quantitative studies that are related to the aspect of science and technology. The focus of MOSAIC is to combine various effective aspects of quantitative skills that will be beneficial for professional lives of students in both science and social sciences [30, 31]. As for statistics, this project focuses in teaching introductory statistics that are highly fused with computing elements in general and RStudio in specific. Additionally, MOSAIC created a built-in $\mathrm{R}$ package, mosaic which provides simplified and structured statistical concepts which are related to descriptive statistics, visualization, modelling, and statistical inference $[31,32]$. The mosaic package helps novice students to deal with difficulties in using RStudio, as it is often regarded as unappealing and inaccessible to them. Students who involved with MOSAIC had confidence in using RStudio, indicating that it is viable to blend computational statistics through RStudio into the syllabus [32].

\subsection{Purpose}

Being the backbone of modern data science, computational statistics is deemed to revamp the learning of statistics to a large extent [8]. Therefore, the objective of this study is to determine the impact of computational statistics approach in an introductory statistics course offered in a Malaysian public university. Basically, we wanted to evaluate the effectiveness of computational statistics approach in learning statistics by assessing and then comparing students' performance.

\section{Methodology}

We employed a pre-, post- and delayed post-test quasiexperimental with control group design in this study. Our sample consists of 100 randomly selected students in one of 
the Malaysian public universities. As our focus was on students from non-technical fields of study, students in our sample were those enrolled in various degree programs such as social science and medical science, taking a compulsory introductory statistics course during their first year. Students were equally separated into two groups, namely computational (followed computational statistics approach in learning statistics, $n=50$ ) and conventional (followed only conventional approach in learning statistics, $n=50$ ). Students from the computational group began to follow the computational statistics approach in learning statistics by joining new tutorial classes (replacing the conventional tutorial) for eight weeks, which were conducted by a group of tutors with innovative teaching methods in statistics. Topics such as descriptive statistics, statistical inference, and linear regression were among the key contents of the tutorial. During each session, the teaching focused on concepts rather than computations, as computation tasks were carried out using RStudio. In addition to that, students were also given some take-home assignments that included computation tasks with empirical datasets.

In order to evaluate the effectiveness of computational statistics approach in the learning process of introductory statistics, students were assessed at three stages: initial, medial and final, respectively. A test was prepared in each stage with five multiple choices and five subjective questions. Statistics instructors obtained past year questions from this introductory statistics course (only questions that are related to the topics included in the tutorial had been chosen). On the other hand, students from the computational group attempted to solve the questions by using RStudio in computer laboratory, while students from conventional group took their tests in the usual manner. Initial assessment was carried out as the pre-test in the first week of semester (before tutorial); while the medial assessment as posttest held on the ninth week (at the end of tutorials); and the final assessment referred as delayed post-test in the fourteenth week (five weeks after tutorials ended). Eventually, students' marks from each stage of assessment (initial, medial, final) were compared between both groups.

\subsection{Statistical Analysis}

To evaluate students' initial performance (before exposed to computational statistics approach), independent samples $t$-test were employed on their initial assessment marks. Next, to measure the effectiveness of computational statistics approach in learning statistics, we performed analysis of covariance on the medial and final assessments' marks with the initial assessment marks as the covariate. In all cases, we were interested in comparing the performance of students from both groups and to determine if there is a statistically significant difference between two groups based on their performance at each stage. In order to evaluate students' improvement from one stage to another, we employed the gain score analysis (analysis of difference score) and computed two new variables as below:

1. medial gain score $=$ medial assessment marks of each student in each group - initial assessment marks of each student in each group
2. final gain score $=$ final assessment marks of each student in each group - medial assessment marks of each student in each group

The analysis of covariance looks for difference in adjusted means of medial and final assessment marks (adjusted for initial assessment marks) [33, 34]. By doing this, we are able to statistically control the initial assessment marks (as confounding variable) which is vital to compare the performance of students in medial and final stage of assessment [34]. Gain score analysis reflects how much knowledge each student has gained from the previous assessment, and this technique is preferred over the repeated measures analysis of variance, particularly to the design of this study [33]. Independent samples $t$-test were used on the newly computed gain variables to see if there is a statistically significant difference in students' improvement in performance based on their gain score from initial to medial; and from medial to final assessments. Although analysis of covariance and gain score analysis are positively correlated and may seem to be similar in reaching a conclusion, however the focus of both analyses is clearly distinct $[34,35]$. The focus of analysis of covariance is the differences between both groups in the medial and final assessment stages while keeping any changes in the initial assessment stage as constant [34, 35]. It does not identify how each group changed from one stage to another completely. Meanwhile, the basis of gain score analysis is the changes that occur from initial to medial stage; and from medial to final stage [34, 35]. This allows us to infer on the improvement rates of each group, i.e. whether a group improved or worsened or showed no improvement within the stages of assessment and compared to the other group in a certain stage [33].

\section{Results}

The mean of initial assessment marks (as in Table 1) for computational group was slightly higher than conventional group, suggesting that statistics knowledge from computational group students might be higher than those in conventional group. However, the independent samples $t$-test $(t(98)=-0.99, p>0.05)$ and the $95 \%$ confidence intervals (CI) for the mean difference revealed that the initial assessment marks of students in both (conventional and computational) groups was not statistically significantly different. Thus, we can infer that initially, students in both groups had the same level of statistics knowledge and performed equally.

In the medial assessment, we can see from Table 2 that the mean of computational group students' marks were higher than that of conventional group students. Based on the analysis of covariance $(F(1,97)=51.81, p<0.05)$, there was a statistically significant difference in the medial assessment marks of both groups. Furthermore, pairwise comparison on the marks and $95 \% \mathrm{CI}$ for the mean difference yielded a significant mean difference of the medial assessment marks between both groups. Hence, it shows that students from computational group performed better in the medial assessment than those from the conventional group. 
Table 1. Independent samples $t$-test on initial assessment scores between conventional and computational groups

\begin{tabular}{|c|c|c|c|c|}
\hline Group & Mean marks & $t$ & Significance & $95 \%$ CI for the mean difference \\
\hline Conventional & 33.00 & -0.99 & 0.33 & $(-15.23,5.23)$ \\
Computational & 35.00 & & & \\
\hline
\end{tabular}

Table 2. Analysis of covariance on medial assessment marks between conventional and computational groups

\begin{tabular}{|c|c|c|c|c|c|}
\hline Group & Adjusted mean marks & Mean difference (Significance) & $95 \% \mathrm{CI}$ for the mean difference & $F$ & Significance \\
\hline $\begin{array}{c}\text { Conventional } \\
\text { Computational }\end{array}$ & $\begin{array}{l}33.28 \\
66.32\end{array}$ & $-33.05(0.00)$ & $(-42.28,-23.81)$ & 51.81 & 0.00 \\
\hline
\end{tabular}

Table 3. Analysis of covariance on final assessment marks between conventional and computational groups

\begin{tabular}{|c|c|c|c|c|c|}
\hline Group & Adjusted mean marks & Mean difference (Significance) & $95 \% \mathrm{CI}$ for the mean difference & $F$ & Significance \\
\hline $\begin{array}{c}\text { Conventional } \\
\text { Computational }\end{array}$ & $\begin{array}{l}53.67 \\
75.65\end{array}$ & $-21.98(0.00)$ & $(-31.92,-12.05)$ & 19.81 & 0.00 \\
\hline
\end{tabular}

Table 4. Independent samples $t$-test on medial gain score (medial - initial) of assessment marks

\begin{tabular}{|c|c|c|c|c|}
\hline Group & Mean gain score & $t$ & Significance & $95 \%$ CI for the mean difference \\
\hline Conventional & -0.68 & -5.48 & 0.00 & $(-41.11,-18.81)$ \\
Computational & 29.28 & & & \\
\hline
\end{tabular}

Table 5. Independent samples $t$-test on final gain score (final - medial) of assessment marks

\begin{tabular}{|c|c|c|c|c|}
\hline Group & Mean gain score & $t$ & Significance & 95\% CI for the mean difference \\
\hline Conventional & 9.24 & -3.03 & 0.00 & $(2.11,22.37)$ \\
Computational & 20.48 & & & \\
\hline
\end{tabular}

From Table 3, we can see that the mean of computational group students' marks were once again higher than conventional group students, for the final assessment. Analysis of covariance $(F(1,97)=19.81, p<0.05)$ indicated that the marks of final assessment were statistically significantly different between both groups. The mean difference and 95\% CI for the mean difference between both groups were also significant. It is clear that computational group students performed better than the conventional group students in their final assessment (the assessment after computational statistics approach learning has ended). This showed that students in computational group were able to sustain their performance from medial assessment to the final assessment to do better than the conventional group students.

As in Table 4, the computational group students had higher mean medial gain score of assessment marks (medial - initial) than conventional group students. The independent samples $t$-test on medial gain score $(t(98)=-5.48, p<0.05)$ and the $95 \%$ CI for the mean difference indicated that the medial gain score between both groups was statistically significantly different. Therefore, we can say that the computational group students had greater and significant improvement in their statistics performance and knowledge level from the initial to medial assessment. Meanwhile, conventional group students showed deteriorating improvement from initial to medial assessment.

The final gain score of assessment marks (final - medial) for computational group students were clearly higher than conventional group students, as displayed in Table 5. The final gain score between both groups was statistically significantly different based on the independent samples $t$-test on final gain score $(t(98)=-3.03, p<0.05)$ and $95 \% \mathrm{CI}$ for the mean difference. Thus, we can say that students in computational group managed to show greater improvement (and sustained their previous improvement) in their statistics performance and knowledge level than conventional group students from the medial to final assessment. Although conventional group students did show some improvement from medial to final assessment, yet their improvement was clearly small in relative to that of computational group students.

\section{Conclusions and Recommendations}

Our findings revealed that students who followed the computational statistics approach in learning statistics performed better than those who used only the conventional approach, when medial and final assessments' marks were being compared. As their initial performance was homogeneous, it shows that statistics knowledge and skills acquisition was effective through the computational statistics approach. Moreover, students in the computational group also showed greater improvement from the initial to medial; and the medial to final stage of assessments. Hence, computational statistics approach was also able to sustain students' interest, performance and knowledge in statistics. Thus, our findings implied that computational statistics approach in the learning process of introductory statistics course exerted a positive impact on students' statistics learning and performance, leading towards effective knowledge and skills acquisition for data science. This study is not without its limitations. Although the existing instruction method may be similar in any higher learning institutions in Malaysia, our sample size is not considerably high enough for any generalization of the findings.

Computational statistics approach offers interesting and cre- 
ative ways to learn statistics. It is notable that this approach incorporates different ways of statistical thinking and reasoning [11]. As such, students will be captivated to be engaged throughout their statistics learning. Ultimately, students will appreciate and apprehend to the overall learning process of statistics. Instructors who have previously taught their courses with less computational statistics approach, may need to consider certain changes in their instruction strategy [29]. For example, although the use of tabulations of critical values of distributions are important for students to understand in-depth the theory and concept of probability in statistics, instructors can minimize the conventional way to read statistical tables by making use of computational tools [7]. Instructors may replace teaching of computational formulas with computation itself by focusing on how to lead to understanding of the computed statistic instead of the computation or derivation steps [7]. Another interesting example will be the statistical inference techniques, a major part of introductory statistics courses that are usually taught as disconnected topics in the conventional approach [36]. This procedure can be enormous to students; and computational tools like RStudio helps to unify statistical inference concepts by one built-in function [17]. This reflects how computational statistics approach can become handy in learning statistics while enabling students to think about the nature of data and encouraging them to carry out exploratory data analysis.

Instructors should take advantage of computational tools to carry out tasks like working with large datasets; creating great visualizations; teaching of resampling or simulation techniques; perform computations efficiently; and concentrate more on conceptual analysis rather than calculations which may not be possible in conventional approach [32]. As most of the proper computational tools (like RStudio) require a fair amount of coding, instructors can use this opportunity to teach coding (an integral element of data science) as a way to introduce or reinforce statistical concepts/ideas especially simulations, that are difficult to convey without computation [37]. Although computational statistics approach has advantages over conventional approach, it is still important to preserve a perfect balance of both approaches to ensure students not to be overloaded, as suggested by the Guidelines for Assessment and Instruction in Statistics Education (GAISE2016) [10]. Introductory statistics is the preliminary course for students across different disciplines to acquire adequate statistical knowledge and skills. The main objective of the course as a precursor to data science must be adhered, which is essentially to gain computing skills to thrive in the world of data.

\section{REFERENCES}

[1] D. Nolan, D. T. Lang. Computing in the statistics curricula, The American Statistician, Vol.64, No.2, 97-107, 2010.

[2] N. J. Horton, B. S. Baumer, H. Wickham. Setting the stage for data science: integration of data management skills in introductory and second courses in statistics, CHANCE,
Vol.28, 40-50, 2015.

[3] N. J. Horton, J. S. Hardin. Teaching the next generation of statistics students to "Think with data": Special issue on statistics and the undergraduate curriculum, The American Statistician, Vol.69, No.4, 259-265, 2015.

[4] J. Ridgway. Implications of the data revolution for statistics education, International Statistical Review, Vol.84, No.3, 528-549, 2015.

[5] N. J. Horton, B. S. Baumer, H. Wickham. Teaching precursors to data science in introductory and second courses in statistics, In: K. Makar, B. deSousa, R. Gould (eds), Sustainability in statistics education, Proceedings of ICOTS 9, International Statistical Institute, Voor-burg, the Netherlands, 2014.

[6] B. Chance, D. Ben-Zvi, J. Garfield, E. Medina. The role of technology in improving student learning of statistics, Technology Innovations in Statistics Education, Vol.1, No.1, Retrieved from https://escholarship.org/uc/item/8sd2t4rr, 2015.

[7] X. Wang, C. Rush, N. J. Horton. Data Visualization on Day One: Bringing Big Ideas into Intro Stats Early and Often, Technology Innovations in Statistics Education, Vol.10, No.1, Retrieved from https://escholarship.org/uc/item/84v3774z, 2017.

[8] J. E. Gentle, W. K. Härdle, Y. Mori. How computational statistics became the backbone of modern data science, In Handbook of Computational Statistics, Springer, Heidelberg, 3-16, 2012.

[9] M. Aliaga, G. W. Cobb, C. Cuff, J. Garfield, R. Gould, R. Lock, T. Moore, A. Rossman, B. Stephenson, J. Utts, P. Velleman, J. Witmer. Guidelines for assessment and instruction in statistics education (GAISE), American Statistical Association, Washington, DC, Retrieved from http://www.amstat.org/education/gaise/GAISECollege, 2005.

[10] R. H. Carver, M. Everson, J. Gabrosek, N. J. Horton, R. H. Lock, M. Mocko, A. Rossman, G. H. Rowell, P. Velleman, J. Witmer, B. Wood. Guidelines for assessment and instruction in statistics education (GAISE), American Statistical Association, Washington, DC, Retrieved from http://www.amstat.org/education/gaise/GAISECollege, 2016.

[11] G. W. Cobb. The introductory statistics course: A Ptolemaic curriculum?, Technology Innovations in Statistics Education, Vol.1, No.1, Retrieved from https://escholarship.org/uc/item/6hb3k0nz, 2012.

[12] W. Finzer. The data science education dilemma, Technology Innovations in Statistics Education, Vol.7, No.1, Retrieved from https://escholarship.org/uc/item/7gv0q9dc, 2013. 
[13] R. Basturk. The effectiveness of computer-assisted instruction in teaching introductory statistics, Journal of Educational Technology \& Society, Vol.8, No.2, 170-178, 2005.

[14] R. Gould. Statistics and the modern student, International Statistical Review, Vol.78, No.2, 297-315, 2010.

[15] G. Grolemund, H. Wickham. A cognitive interpretation of data analysis, International Statistical Review, Vol.82, No.2, 184-204, 2014.

[16] A. McNamara. On the State of Computing in Statistics Education: Tools for Learning and for Doing, arXiv preprint arXiv: 1610.00984, 2016.

[17] J. Baglin. Applying a theoretical model for explaining the development of technological skills in statistics education, Technology Innovations in Statistics Education, Vol.7, No.1, Retrieved from https://escholarship.org/uc/item/8w97p75s, 2013.

[18] M. Meletiou-Mavrotheris, C. Lee, R. T. Fouladi. Introductory statistics, college student attitudes and knowledge-a qualitative analysis of the impact of technology-based instruction, International Journal of Mathematical Education in Science and Technology, Vol.38, No.1, 65-83, 2007.

[19] D. Ben-Zvi D. Toward understanding the role of technological tools in statistical learning, Mathematical thinking and learning, Vol.2, No.1-2, 127-155, 2000.

[20] M. Alias. Integrating technology into classroom instructions for reduced misconceptions in statistics, International Electronic Journal of Mathematics Education, Vol.4, No.2, Retrieved from http://eprints.uthm.edu.my/1850, 2009.

[21] N. J. Horton, E. R. Brown, L. Qian. Use of R as a toolbox for mathematical statistics exploration, The American Statistician, Vol.58, No.4, 343-357, 2004.

[22] R Core Team. R: A language and environment for statistical computing, $\mathrm{R}$ Foundation for Statistical Computing, https://www.r-project.org/, 2016.

[23] J. Verzani. Using R for introductory statistics, CRC Press, New York, NY, 2014.

[24] R. E. Schumacker. Learning statistics using R, Sage Publications, Los Angeles, CA, 2014.

[25] RStudio Team. RStudio: Integrated Development for R, http://www.rstudio.com/products/rstudio/, 2014.
[26] D. Navarro D. Learning Statistics with R: A Tutorial for Psychology Students and Other Beginners: Version 0.5, Retrieved from https://learningstatisticswithr.com/, 2015.

[27] P. Dalgaard. Introductory statistics with R, Springer-Verlag, New York, NY, 2008.

[28] N. J. Horton, K. Kleinman. Using R for data management, statistical analysis, and graphics, CRC Press, New York, NY, 2010.

[29] A. J. R. Godfrey. Statistical Software from a Blind Person's Perspective, R Journal, Vol.5, No.7, 73-79, 2013.

[30] R. Pruim, N. J. Horton, D. Kaplan. Start teaching with R, Project MOSAIC, Retrieved from http://mosaic-web.org/go/MasterStarting.pdf, 2014.

[31] R. Pruim, D. Kaplan, N. J. Horton. mosaic: project MOSAIC statistics and mathematics teaching utilities, $\mathrm{R}$ package version 0.14.4, https://cran.r-project.org/web/packages/mosaic/, 2016.

[32] R. Pruim, D. Kaplan, N. J. Horton. The mosaic Package: Helping Students to 'Think with Data' Using R, R Journal, Vol.9, No.1, 77-102, 2017.

[33] D. M. Dimitrov, P. D. Rumrill Jr. Pretest-posttest designs and measurement of change, Work, Vol.20, No.2, 159-165, 2003.

[34] T. R. Knapp, W. D. Schafe. From gain score t to ANCOVA F (and vice versa), Practical Assessment, Research \& Evaluation, Vol.14, No.6, 1-7, 2009.

[35] L. A. Becker. Analysis of pretest and posttest scores with gain scores and repeated measures, FrontPage Workshop Lecture Notes, Retrieved from http://www. uccs. edu/lbecker/gainscore.html, 2000.

[36] C. J. Wild, M. Pfannkuch, M. Regan, N. J. Horton. Towards more accessible conceptions of statistical inference, Journal of the Royal Statistical Society: Series A (Statistics in Society), Vol.174, No.2, 247-295, 2011.

[37] R. H. Carver. Introductory Statistics Unconstrained by Computability: A New Cobb Salad, Technology Innovations in Statistics Education, Vol.5, No.1, Retrieved from https://escholarship.org/uc/item/81d8c04j, 2011. 